\title{
ADRC based control for a class of input time delay systems
}

\author{
Dongyang Zhang, Xiaolan Yao*, Qinghe Wu, and Zhuoyue Song \\ School of Automation, Beijing Institute of Technology, Beijing 100081, China
}

\begin{abstract}
This paper is concerned with the control design and the theoretical analysis for a class of input time-delay systems with stable, critical stable or unstable poles. In order to overcome the time delay, a novel feed-forward compensation active disturbance rejection control (FFC-ADRC) approach is proposed. It combines advantages of the Smith predictor and the traditional active disturbance rejection control (ADRC). The tracking differentiator (TD) is designed to predict the control signal, which adds an anticipatory control to the control signal and allows a higher observer bandwidth to obtain better disturbance rejection. The modified extended state observer (ESO) is designed to estimate both system states and the total disturbances (internal disturbance, uncertainties and delayed disturbance). Then the Lyapunov theory and the theory of the input-output stability are applied to prove the asymptotic stability of the closed-loop control system. Finally, numerical simulations show the effectiveness and practicality of the proposed design.
\end{abstract}

Keywords: time-delay system, feed-forward compensation active disturbance rejection control (FFC-ADRC), tracking differentiator (TD), Lyapunov theory, bound-input-bound-output (BIBO) stability.

DOI: $10.21629 / J S E E .2017 .06 .19$

\section{Introduction}

Most industrial process, e.g. network [1], aircraft [2] and especially process control [3], are often treated as either first order plus time delay (FOPTD) systems or second order plus time delay (SOPTD) systems to simplify the controller design. The time delay which is also known as the dead-time is generally produced by the actual delays (e.g. the delays in actuators and sensors) or an approximation of a higher order dynamic to a lower order one [4]. The control design for a time-delay system is very challenging due to the fact that the time delay introduces extra phase lag leading to reduce the stability margin and even put a stringent limit on the bandwidth [5]. The achievable closedloop bandwidth is normally limited to $1 / \tau$, where $\tau$ is the time delay [6]. Therefore, a specific control method must

\footnotetext{
Manuscript received July 18, 2016.

*Corresponding author.

This work was supported by the National Natural Science Foundation of China (61304026).
}

be developed to eliminate the influence brought by the time delay.

Over the years, many methods have been developed for the control of time-delay systems effectively, such as the proportional-integral-derivative (PID) control, Smith predictor (SP) control, $\mathrm{H} \infty$ control and their modified methods [7-23]. It should be noted that the mainstream approach in real engineering is the PID control $[7-9,19]$. However, there is not much theory dealing with PID design, so most of industrial PID designs are carried out by only using empirical techniques. Thus, mathematically elegant and sophisticated theories developed in the context of modern optimal control cannot be applied to them. And the performance of PID will be worse with the increase of the time delay [16]. Based on that, the SP control was presented by Smith [12] to improve the performance for systems with time delay. It is one of the most popular timedelay compensation methods. It can increase the closedloop bandwidth in theory via removing the time delay out from the closed loop. However, this method needs an accurate system model to achieve good performance. And it is sensitive to model uncertainties and cannot deal with the unstable time-delay system. Although some modifications $[14,22]$ have been done based on the original SP scheme to improve the performance, model uncertainties remain to be a primary cause of instability in modified SP methods. Furthermore, a common characteristic of aforementioned methods is the poor anti-disturbance ability. And many existing methods failed to provide an on-line tuning rule of controller parameters to cope with the system uncertainty.

To further enhance the control capability of the reference input tracking and the disturbance rejection for timedelay systems, this paper presents a modified active disturbance rejection control (ADRC) method. ADRC proposed by Han [24] has become quite an attractive methodology for applied researchers. It possesses many particular advantages, i.e. the ability of dealing with a vast range of uncertainties, improving transient response, implementing easily, saving energy, and so on [25]. Nowadays, ADRC has played an important role in a lot of fields because of 
its unique advantages, such as those proposed in [26-28]. Then some researches have proved its convergence performance [29,30] and achieved its tuning rules [31] to facilitate the implementation of the ADRC method. In recent years, applications of ADRC in time-delay systems have been studied [32-36]. The original ADRC scheme deals with the time-delay problem by two approaches [32-34], i.e. ignoring the time delay and approximating the time delay via a first-order inertia unit. The former can avoid the order increase; however, the expense of the extended state observer (ESO) is the heavy burden. As for the latter, the limitation of the estimate ability of ESO makes the original ADRC feasible only when the time-delay is small. Other methods try to synchronize the system output and control input by adding a predictor before ESO [35]. However, the theory analysis of these methods lagged behind applications for quite some time and lacked of general conclusions. That's why dealing with the time delay is always a fundamental problem in the control design. Therefore, this paper will focus on a modified ADRC design and its theoretical analysis for a class of input time-delay systems with stable, critical stable or unstable poles.

This paper proposes a novel feed-forward compensation active disturbance rejection control (FFC-ADRC) method to reduce the influence of the time delay. An anticipatory control is added to the control signal before it is input into the system and ESO, which can synchronize input signals of ESO such that providing meaningful estimations of time-delay system states and the delayed disturbance. The modified control design is simple, and it enhances the control performance by increasing the achievable observer bandwidth which is the key for an accurate estimation of the total disturbance. Then the superiority analysis about the FFC-ADRC and the stability of the closed-loop system are carried out. With an appropriate tuning, the proposed method provides a unified solution to a class of input time-delay systems (with stable, critical stable, or unstable poles).

This paper is organized as follows. In Section 2, the traditional ADRC design is first introduced followed by the proposed modification. In Section 3, the characteristics of FFC-ADRC and the stability of the closed-loop control system are discussed in detail. Then the procedure of parameter tuning is analyzed. In Section 4, numerical simulations show the improvement of FFC-ADRC compared with the existing method. The conclusions are given in Section 5.

\section{Derivation of the method}

\subsection{Analysis of time-delay systems}

Generally, a processing system with time delay can be pre- sented as an FOPTD system or an SOPTD system:

$$
\begin{gathered}
G_{\text {FOPTD }}(s)=\frac{b}{s+a} \mathrm{e}^{-\tau s} \\
G_{\text {SOPTD }}(s)=\frac{b}{s^{2}+a_{1} s+a_{0}} \mathrm{e}^{-\tau s}
\end{gathered}
$$

where $\tau$ is the delay time of the system. In most cases, $a>0, a_{1}>0$ and $a_{0}>0$, i.e. all poles are at the left part plane. In the proposed design, we will also consider the other two cases: (i) with $a=0, a_{0}=0$, systems become integral processes with the time delay; (ii) with $a<0$ and $a_{1}<0$ or $a_{0}<0$, systems become unstable, which make the problem even more challenging.

For the closed-loop control system in Fig. 1, we can note that if the controller is designed when ignoring the time delay, the control input $u(t)$ can only have effect on the output signal after $\tau$ moment; furthermore, the response of the disturbance $d(t)$ injected at time $t$ will be fed back to the controller at time $t+\tau$, and what worse is that the control signal will affect the output after another $\tau$ time. Therefore, the real-time control performance is poor in systems with time delay. This problem is the focus of this paper.

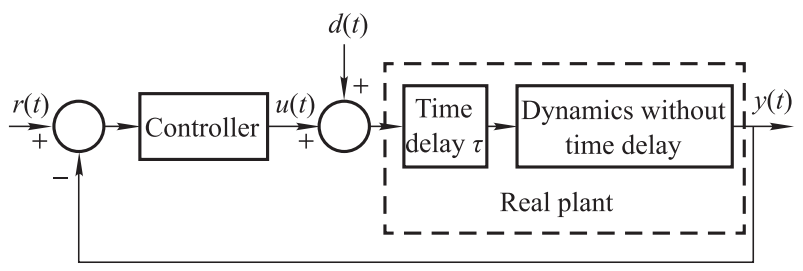

Fig. 1 Closed-loop control system with time delay

In system (1) and (2), there is an infinite dimensional factor $\mathrm{e}^{-\tau s}=\frac{1}{\sum_{i=0}^{\infty} l_{i} s^{i}}, \quad l_{i}=\frac{1}{i !} \tau^{i}$. When $\tau$ is small enough and $i$ is large enough, the expansion of the infinite dimensional factor is a rapid convergent series. In another word, high-order term round towards zero and it can be equal to a finite dimensional equation. Thus, one can obtain that the time delay system can be controlled by a finite dimensional controller.

The difficulty of control for the input time-delay system can be described as $\theta=\tau / T$, where $T$ is the time constant of the system [16]. With the increasing of $\theta$, the control difficulty is more serious. If $\theta>0.5$, the system is called as "large time-delay system", or else "universal time-delay system".

\subsection{Traditional ADRC method}

Generally, a nonlinear uncertain system can be described as

$$
\begin{gathered}
y(t)^{(n)}=d(t)+f\left(y(t), \ldots, y(t)^{n-1}, t\right)+b u(t), \\
t>t_{0}
\end{gathered}
$$


where $u(t)$ and $y(t)$ are the control input and the output, respectively; $d(t)$ is the external disturbance; $f\left(y(t), \ldots, y(t)^{n-1}, t\right)$ is internal unknown dynamics.

In order to estimate the total disturbance, we define an extended state variable, $x_{n+1}=d(t)+f(y(t), \ldots$, $\left.y(t)^{n-1}, t\right)+(b-\bar{b}) u(t) \triangleq p\left(y(t), \ldots, y(t)^{n-1}, t\right)$, where $\bar{b}$ is the estimation of $b$ (when $b$ is uncertain), and $p\left(y(t), \ldots, y(t)^{n-1}, t\right)$ is the total disturbance. Then let $x_{1}=y(t), x_{2}=\dot{y}(t), \ldots, x_{n}=y(t)^{n-1}$. Assuming the total disturbance $p(\boldsymbol{X}, t)$ is differentiable, the extended state space expression of the system (3) is given by

$$
\left\{\begin{array}{l}
\dot{\boldsymbol{X}}=\boldsymbol{A}_{g} \boldsymbol{X}+\bar{b} \boldsymbol{B}_{g} u(t)+\boldsymbol{E}_{g} h \\
y=x_{1}
\end{array}\right.
$$

where $\boldsymbol{A}_{g}=\left[\begin{array}{cccc}0 & 1 & 0 & 0 \\ \vdots & \vdots & \vdots & \vdots \\ 0 & 0 & 0 & 1 \\ 0 & 0 & 0 & 0\end{array}\right], \boldsymbol{B}_{g}=\left[\begin{array}{c}0 \\ \vdots \\ 1 \\ 0\end{array}\right], \quad \boldsymbol{X}=$ $\left[\begin{array}{c}x_{1} \\ \vdots \\ x_{n} \\ x_{n+1}\end{array}\right], \boldsymbol{E}_{g}=\left[\begin{array}{c}0 \\ \vdots \\ 0 \\ 1\end{array}\right], h=\dot{p}(\boldsymbol{X}, t)$.

ESO is the core of ADRC and used to estimate not only system states but also the total disturbance (including the external and internal disturbance); the control input signal $u(t)$, and the system output $y(t)$, are inputs of ESO. The linear form of ESO is given by

$$
\left\{\begin{array}{c}
\dot{z}_{1}=z_{2}+\beta_{1}\left(y-z_{1}\right) \\
\vdots \\
\dot{z}_{i}=z_{i+1}+\beta_{i}\left(y-z_{1}\right) \\
\vdots \\
\dot{z}_{n}=z_{n+1}+\beta_{n}\left(y-z_{1}\right)+\bar{b} u(t) \\
\dot{z}_{n+1}=\beta_{n+1}\left(y-z_{1}\right)
\end{array}\right.
$$

where $\beta_{i}$ are design parameters of ESO. Parameters $\beta_{i}(i=1,2, \ldots, n+1)$ are required to be well tuned such that state variables of ESO, $z_{i}(i=1,2, \ldots, n+1)$ can be seen as estimations of $x_{i}(i=1,2, \ldots, n+1)$.

The state error feedback and the disturbance compensation are designed as

$$
\begin{gathered}
u_{0}=k_{1}\left(r(t)-z_{1}\right)-k_{2} z_{2}-\cdots-k_{n} z_{n} \\
u(t)=\frac{u_{0}-z_{n+1}}{\bar{b}}
\end{gathered}
$$

where $r(t)$ is the reference signal; $k_{i}$ is the controller parameter, $i=1,2, \ldots, n$.

As for a typical FOPTD (1), its extended state space expression is given by

$$
\left\{\begin{array}{l}
\dot{\boldsymbol{X}}(t)=\boldsymbol{A} \boldsymbol{X}(t)+\bar{b} \boldsymbol{B} u(t-\tau)+\boldsymbol{E} h \\
y(t)=\boldsymbol{C} \boldsymbol{X}(t)
\end{array}\right.
$$

where $\boldsymbol{A}=\left[\begin{array}{ll}0 & 1 \\ 0 & 0\end{array}\right], \boldsymbol{B}=\left[\begin{array}{l}1 \\ 0\end{array}\right], \boldsymbol{C}=\left[\begin{array}{l}1 \\ 0\end{array}\right]^{\mathrm{T}}, \boldsymbol{E}=\left[\begin{array}{l}0 \\ 1\end{array}\right]$, $\boldsymbol{X}=\left[\begin{array}{l}x_{1} \\ x_{2}\end{array}\right]=\left[\begin{array}{l}y \\ p\end{array}\right], p(\boldsymbol{X}, t)$ is the total disturbance, and $h$ is the differential of $p(\boldsymbol{X}, t)$.

Then based on the design of ADRC above, the firstorder ADRC is designed. Let $\boldsymbol{L}=\left[\begin{array}{ll}\beta_{1} & \beta_{2}\end{array}\right]^{\mathrm{T}}$, and the output estimate of ESO is $\bar{y}(t)=\boldsymbol{C} \boldsymbol{Z}(t), \boldsymbol{Z}(t)=\left[\begin{array}{ll}z_{1} & z_{2}\end{array}\right]^{\mathrm{T}}$. The control signal is given by

$$
u=\frac{1}{\bar{b}} \boldsymbol{F} \boldsymbol{Z}(t)+\frac{k_{1}}{\bar{b}} r(t)
$$

where $\boldsymbol{F}=\left[\begin{array}{ll}-k_{1} & -1\end{array}\right]$. Substituting (9) into the extended system (8) and the traditional ESO simultaneously, yields that

$$
\begin{gathered}
\dot{\boldsymbol{X}}(t)=\boldsymbol{A} \boldsymbol{X}(t)+\boldsymbol{B} \boldsymbol{F} \boldsymbol{Z}(t-\tau)+k_{1} \boldsymbol{B} r(t-\tau)+\boldsymbol{E} h \\
\dot{\boldsymbol{Z}}(t)=\boldsymbol{A} \boldsymbol{Z}(t)+\boldsymbol{B} \boldsymbol{F} \boldsymbol{Z}(t)+\boldsymbol{B} k_{1} r(t)+ \\
\boldsymbol{L} \boldsymbol{C}(\boldsymbol{X}(t)-\boldsymbol{Z}(t)) .
\end{gathered}
$$

Defining $\boldsymbol{e}(t)=\boldsymbol{X}(t)-\boldsymbol{Z}(t)$, the observer error of the traditional ESO can be expressed as

$$
\begin{gathered}
\dot{\boldsymbol{e}}(t)=(\boldsymbol{A}-\boldsymbol{L} \boldsymbol{C}) \boldsymbol{e}(t)-\boldsymbol{B} \boldsymbol{F} \boldsymbol{e}(t-\tau)+ \\
\boldsymbol{B} \boldsymbol{F} \boldsymbol{e}(t)+\boldsymbol{E} h+\boldsymbol{B} \boldsymbol{F} \boldsymbol{X}(t-\tau)- \\
\boldsymbol{B} \boldsymbol{F} \boldsymbol{X}(t)+\boldsymbol{B} k_{1} r(t-\tau)-\boldsymbol{B} k_{1} r(t) .
\end{gathered}
$$

According to (12), $\boldsymbol{e}(t)$ relates to the variable $\boldsymbol{X}(t)$ and $r(t)$ due to the time delay $\tau$. Therefore, it is difficult to obtain $e(t) \rightarrow 0$ via adjusting parameters of ESO, that is, it may lead to ESO instability. Similarly, the SOPTD system has the same problem.

Thus, as for time delay systems, because the output estimation of the traditional ESO and the system output are not synchronized, it may lead to the instability of ESO.

To solve this problem, we should modify the traditional ADRC to eliminate the time misalign between the control signal $u(t)$ and output signal $y(t)$. In this paper, a novel predictor based on an SP is proposed to address this problem.

\subsection{FFC-ADRC method}

Starting from the essence of SP, as shown in Fig. 2, $G(s)\left(1-\mathrm{e}^{-\tau s}\right)$ is paralleled with the system dynamics as a feed-forward loop.

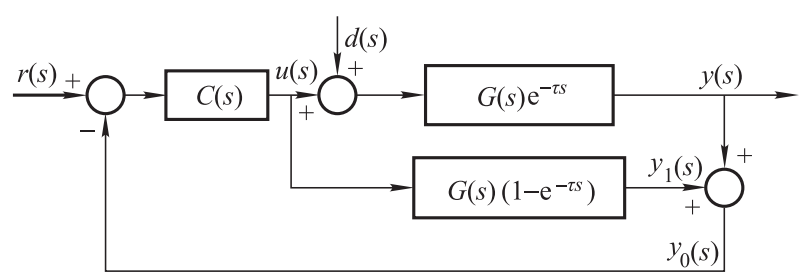

Fig. 2 SP 
However, the SP method needs an accurate model. Based on that reason, we make some modifications. The relationship between $u(s)$ and $y_{0}(s)$ in Fig. 2 is given by

$$
\begin{gathered}
y_{0}(s)=y(s)+y_{1}(s)= \\
G(s) \mathrm{e}^{-\tau s} u(s)+G(s)\left(1-\mathrm{e}^{-\tau s}\right) u(s)= \\
G(s) \mathrm{e}^{-\tau s}\left(1+\frac{1-\mathrm{e}^{-\tau s}}{\mathrm{e}^{-\tau s}}\right) u(s)= \\
G(s) \mathrm{e}^{-\tau s} u(s) \mathrm{e}^{\tau s} .
\end{gathered}
$$

According to the taylor series expansion $\mathrm{e}^{x}=1+x+$ $\frac{1}{2 !} x^{2}+\cdots+\frac{1}{n !} x^{n}+\cdots, 1+\tau s$ is taken as the approximation of $\mathrm{e}^{\tau s}[36]$, then

$$
y_{0}(s)=G(s) \mathrm{e}^{-\tau s} u(s)(1+\tau s)
$$

where $u(s)(1+\tau s)$ is viewed as a whole, that is to say, the predicted signal $y_{0}(s)$ can be derived via $u(s)(1+\tau s)$ (i.e. $u(s)$ and $s u(s)$ ). As mentioned before, the tracking differentiator (TD) can provide the differential of a signal by dealing with the signal itself. Therefore, TD is proposed to obtain the prediction of signal to realize the function of the phase advance, as shown in Fig. 3.

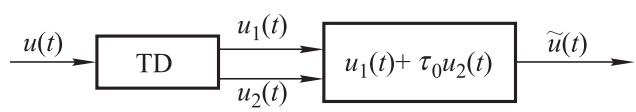

Fig. 3 Modification of the control signal via TD

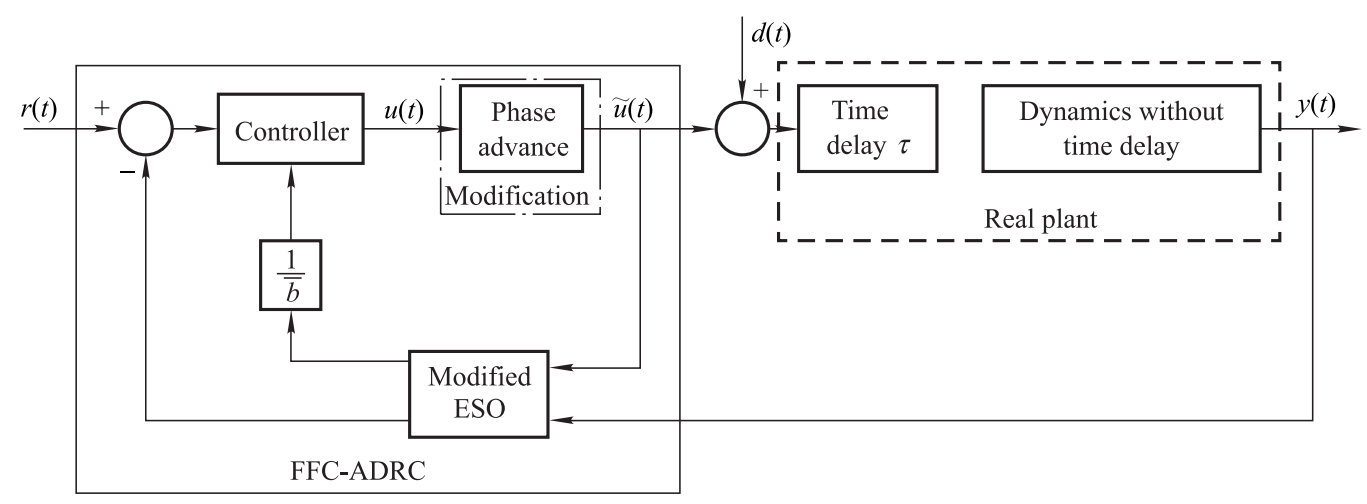

Fig. 4 Schematic of FFC-ADRC

With this modification, the output signal of the closeloop system is synchronous with the control signal, which guarantees ESO and the closed-loop system stable. We will prove it in Section 3.

\section{Characteristics analysis of closed-loop system}

The convergence analysis of ESO and the stability of the closed-loop system are the prerequisite to apply the modi-
In Fig. 3, the discrete-time form of a TD is shown below

$$
\left\{\begin{array}{l}
e_{\mathrm{TD}}=u_{1}(k)-u(k) \\
u_{1}(k+1)=u_{1}(k)+h_{1} u_{2}(k) \\
u_{2}(k+1)=u_{2}(k)+h_{1} \text { fhan }\left(e_{\mathrm{TD}}, u_{2}, r, h_{1}\right)
\end{array}\right.
$$

where $r_{1}$ is the convergence factor, $h_{1}$ is the sample step, $u(k)$ is the control input signal; $u_{1}(k)$ is the tracking signal of $u(k) ; u_{2}(k)$ is the differential signal of $u_{1}(k) ; e_{\mathrm{TD}}$ is the tracking error of TD and fhan [24] is defined as

$$
\begin{gathered}
\operatorname{fhan}\left(e_{\mathrm{TD}}, u_{2}, r_{1}, h_{1}\right)=\left\{\begin{array}{l}
-r_{1} \operatorname{sign}(a), \quad|a|>d \\
-r_{1} a / d, \quad|a| \leqslant d
\end{array}\right. \\
d=r_{1} h_{1}, d_{0}=h_{1} d, y=e_{\mathrm{TD}}+h_{1} u_{2}, \\
a_{0}=\sqrt{d^{2}+8 r_{1}|y|}, \\
a=\left\{\begin{array}{l}
u_{2}+\operatorname{sign}(y)\left(a_{0}-d\right) / 2, \quad|y|>d_{0} \\
u_{2}+y / h_{1}, \quad|y| \leqslant d_{0}
\end{array}\right.
\end{gathered}
$$

With this modification, FFC-ADRC is shown in Fig. 4. Then every part in the closed-loop system is given by

$$
\begin{gathered}
\left\{\begin{array}{l}
\dot{\boldsymbol{X}}(t)=\boldsymbol{A} \boldsymbol{X}(t)+\bar{b} \boldsymbol{B} \tilde{u}(t-\tau)+\boldsymbol{E} h \\
y(t)=\boldsymbol{C} \boldsymbol{X}(t)
\end{array}\right. \\
\left\{\begin{array}{l}
\dot{\boldsymbol{Z}}(t)=\boldsymbol{A} \boldsymbol{Z}(t)+\bar{b} \boldsymbol{B} \tilde{u}\left(t-\tau_{0}\right)+\boldsymbol{L}(y(t)-\bar{y}(t)) \\
\bar{y}(t)=\boldsymbol{C} \boldsymbol{Z}(t)
\end{array}\right. \\
\tilde{u}(t)=u(t)+\tau_{0} \dot{u}(t) \\
u(t)=\frac{1}{\bar{b}} \boldsymbol{F} \boldsymbol{Z}(t)+\frac{1}{\bar{b}} k_{1} r(t)
\end{gathered}
$$

where $\tau_{0}$ is the estimate value of $\tau$.

fied ADRC. And the parameter tuning is the key part for the performance of FFC-ADRC. In this section, both the convergence analysis of the modified ESO and the stability of the closed-loop system are verified; and then the parameter tuning is analyzed in order to provide an excellent performance for time-delay systems.

\subsection{FFC-ADRC analysis}

For FOPTD system, substituting (18) and (19) into (16) 
and (17) yields

$$
\begin{gathered}
\tilde{u}(t)=\frac{1}{\bar{b}} \boldsymbol{F} \boldsymbol{Z}(t)+\frac{k_{1}}{\bar{b}} r(t)+ \\
\tau_{0} \frac{1}{\bar{b}} \boldsymbol{F} \dot{\boldsymbol{Z}}(t)+\tau_{0} \frac{k_{1}}{\bar{b}} \dot{r}(t) \\
\dot{\boldsymbol{X}}(t)=\boldsymbol{A} \boldsymbol{X}(t)+\boldsymbol{B} \boldsymbol{F} \boldsymbol{Z}(t-\tau)+ \\
\boldsymbol{B} k_{1} r(t-\tau)+\boldsymbol{B} \boldsymbol{F} \tau_{0} \dot{\boldsymbol{Z}}(t-\tau)+ \\
\boldsymbol{B} k_{1} \tau_{0} \dot{r}(t-\tau)+\boldsymbol{E} h \\
\dot{\boldsymbol{Z}}(t)=\boldsymbol{A} \boldsymbol{Z}(t)+\boldsymbol{B} \boldsymbol{F} \boldsymbol{Z}\left(t-\tau_{0}\right)+ \\
\boldsymbol{B} k_{1} r\left(t-\tau_{0}\right)+\boldsymbol{B} \boldsymbol{F} \tau_{0} \dot{Z}\left(t-\tau_{0}\right)+ \\
\boldsymbol{B} k_{1} \tau_{0} \dot{r}\left(t-\tau_{0}\right)+\boldsymbol{L} \boldsymbol{C}(\boldsymbol{X}(t)-\boldsymbol{Z}(t)) .
\end{gathered}
$$

Assuming $\tau_{0}=\tau$, the observer error dynamic of the modified ESO can be obtained via (21) and (22):

$$
\begin{gathered}
\dot{\boldsymbol{e}}(t)=\dot{\boldsymbol{X}}(t)-\dot{\boldsymbol{Z}}(t)= \\
(\boldsymbol{A}-\boldsymbol{L} \boldsymbol{C}) \boldsymbol{e}(t)+\boldsymbol{E} h .
\end{gathered}
$$

Since $\boldsymbol{N}=\left[\begin{array}{c}\boldsymbol{C} \\ \boldsymbol{C A}\end{array}\right]=\left[\begin{array}{ll}1 & 0 \\ 0 & 1\end{array}\right]$ is of full rank, $(\boldsymbol{C}, \boldsymbol{A})$ is observable, and poles of (23) can be placed at anywhere by adjusting parameter matrix $\boldsymbol{L}$. More straightforward to say instead, the characteristic equation of (23) is $\|s \boldsymbol{I}-\boldsymbol{A}+\boldsymbol{L} \boldsymbol{C}\|=s^{2}+\beta_{1} s+\beta_{2}$, and the root of characteristic equation can be set at left half plane via tuning parameter matrix $\boldsymbol{L}$. Therefore, the estimate error $\boldsymbol{e}(t)$ of ESO can converge to zero with proper parameters, and ESO will be stable after some time. In this way, $\boldsymbol{Z}(t)$ can estimate $\boldsymbol{X}(t)$ accurately and rapidly.

In the simulation section, $\|s \boldsymbol{I}-\boldsymbol{A}+\boldsymbol{L} \boldsymbol{C}\|=s^{2}+\beta_{1} s+$ $\beta_{2}=\left(s+\omega_{o}\right)^{2}$ is guaranteed by making $\beta_{1}=2 \omega_{0}, \beta_{2}=$ $\omega_{0}^{2}$, where $\omega_{0}$ is the observer bandwidth.

For $\tau_{0}=\tau,(21)$ can be rewritten as

$$
\begin{gathered}
\dot{\boldsymbol{X}}(t)=\boldsymbol{A} \boldsymbol{X}(t)+\boldsymbol{B} \boldsymbol{F} \boldsymbol{Z}\left(t-\tau_{0}\right)+ \\
\boldsymbol{B} k_{1} r\left(t-\tau_{0}\right)+\boldsymbol{B} \boldsymbol{F} \tau_{0} \dot{\boldsymbol{Z}}\left(t-\tau_{0}\right)+ \\
\boldsymbol{B} k_{1} \tau_{0} \dot{r}\left(t-\tau_{0}\right)+\boldsymbol{E} h .
\end{gathered}
$$

According to the taylor series expansion, i.e. $f(x)=f(x-$ $\left.x_{0}\right)+x_{0} \dot{f}\left(x-x_{0}\right)+o\left(x^{2}\right)$, the terms $\boldsymbol{Z}\left(t-\tau_{0}\right)+\tau_{0} \dot{\boldsymbol{Z}}\left(t-\tau_{0}\right)$ and $r\left(t-\tau_{0}\right)+\tau_{0} \dot{r}\left(t-\tau_{0}\right)$ are approximated as $\boldsymbol{Z}(t)$ and $r(t)$, therefore one can obtain that

$$
\dot{\boldsymbol{X}}(t)=\boldsymbol{A} \boldsymbol{X}(t)+\boldsymbol{B} \boldsymbol{F} \boldsymbol{Z}(t)+\boldsymbol{B} k_{1} r(t)+\boldsymbol{E} h .
$$

It is exactly the system when (9) has no time delay. Therefore, FFC-ADRC can accurately reject the influence of the time delay and improve the control performance.
We have proved that the modified ESO can estimate system states and the total disturbance accurately, i.e. $\boldsymbol{e}(t)=$ $0, t>t_{1}$, so in (25), $\boldsymbol{Z}(t)$ can be replaced by $\boldsymbol{X}(t)$, then the frequency domain expression of the expected closedloop system is given by

$$
\begin{aligned}
& G(s)=\frac{y(s)}{r(s)}=\frac{\boldsymbol{C} \boldsymbol{X}(s)}{r(s)}= \\
& \boldsymbol{C}(s \boldsymbol{I}-\boldsymbol{A}-\boldsymbol{B} \boldsymbol{F})^{-1} \boldsymbol{B} k_{1} .
\end{aligned}
$$

The time-delay term is eliminated absolutely in (26). Therefore, FFC-ADRC also has an advantage in reducing the phase lag. That is, FFC-ADRC can increase the phase margin so that the larger controller bandwidth is allowed to make a better performance.

Remark 1 For SOPTD systems, it can be analyzed via the same method. Yet because of limitations, we will not analyze it here.

\subsection{Stability analysis of closed-loop system}

The stability analysis of the proposed design for time-delay systems will be proved by using Lyapunov theory and the theory input-output stability in this section.

As for an FOPTD system, applying FFC-ADRC design and combining (1) with (20), the differential equation of the output signal is given by

$$
\begin{gathered}
\dot{y}(t)=-a y(t)+b \tilde{u}(t-\tau)= \\
-a y(t)+\frac{b}{\bar{b}} k_{1} z_{1}(t-\tau)- \\
\frac{b}{\bar{b}} z_{2}(t-\tau)+\frac{b}{\bar{b}} k_{1} r(t-\tau)- \\
\frac{b}{\bar{b}} k_{1} \tau_{0} \dot{z}_{1}(t-\tau)- \\
\frac{b}{\bar{b}} \tau_{0} \dot{z}_{2}(t-\tau)+\frac{b}{\bar{b}} k_{1} \tau_{0} \dot{r}(t-\tau)= \\
-a y(t)-\frac{b}{\bar{b}} k_{1}\left(z_{1}(t-\tau)+\right. \\
\left.\tau_{0} \dot{z}_{1}(t-\tau)\right)-\frac{b}{\bar{b}}\left(z_{2}(t-\tau)+\right. \\
\left.\tau_{0} \dot{z}_{2}(t-\tau)\right)+ \\
\frac{b}{\bar{b}} k_{1}\left(r(t-\tau)+\tau_{0} \dot{r}(t-\tau)\right)= \\
-a y(t)-\frac{b}{\bar{b}} k_{1} z_{1}(t)- \\
\overline{\bar{b}} z_{2}(t)+\frac{b}{\bar{b}} k_{1} r(t) .
\end{gathered}
$$

From (22), estimate states of ESO is given by

$$
\begin{gathered}
\dot{z}_{1}(t)=z_{2}(t)-k_{1} z_{1}\left(t-\tau_{0}\right)- \\
z_{2}\left(t-\tau_{0}\right)+k_{1} r\left(t-\tau_{0}\right)-
\end{gathered}
$$




$$
\begin{gathered}
k_{1} \tau_{0} \dot{z}_{1}\left(t-\tau_{0}\right)-\tau_{0} \dot{z}_{2}\left(t-\tau_{0}\right)+ \\
k_{1} \tau_{0} \dot{r}\left(t-\tau_{0}\right)+\beta_{1} x_{1}(t)-\beta_{1} z_{1}(t)= \\
z_{2}(t)-k_{1}\left(z_{1}\left(t-\tau_{0}\right)+\right. \\
\left.\tau_{0} \dot{z}_{1}\left(t-\tau_{0}\right)\right)-\left(z_{2}\left(t-\tau_{0}\right)+\right. \\
\left.\tau_{0} \dot{z}_{2}\left(t-\tau_{0}\right)\right)+k_{1}\left(r\left(t-\tau_{0}\right)+\right. \\
\left.\tau_{0} \dot{r}\left(t-\tau_{0}\right)\right)+\beta_{1} x_{1}(t)-\beta_{1} z_{1}(t)= \\
\beta_{1} x_{1}(t)-\left(k_{1}+\beta_{1}\right) z_{1}(t)+k_{1} r(t) \\
\dot{z}_{2}(t)=\beta_{2}\left(x_{1}(t)-z_{1}(t)\right)= \\
\beta_{2} x_{1}(t)-\beta_{2} z_{1}(t) .
\end{gathered}
$$

Then the closed-loop system controlled by FFC-ARDC can be rewritten as

$$
\left\{\begin{array}{l}
\dot{\boldsymbol{\Gamma}}(t)=\boldsymbol{A}_{\boldsymbol{\Gamma}} \boldsymbol{\Gamma}(t)+\boldsymbol{B}_{\boldsymbol{\Gamma}} r(t) \\
y_{\boldsymbol{\Gamma}}(t)=\boldsymbol{C}_{\boldsymbol{\Gamma}} \boldsymbol{\Gamma}(t)
\end{array}\right.
$$

where

$$
\begin{gathered}
\boldsymbol{\Gamma}=\left[\begin{array}{c}
y \\
z_{1} \\
z_{2}
\end{array}\right], \quad \boldsymbol{A}_{\boldsymbol{\Gamma}}=\left[\begin{array}{ccc}
-a & -\frac{k_{1} b}{\bar{b}} & -\frac{b}{\bar{b}} \\
\beta_{1} & -k_{1}-\beta_{1} & 0 \\
\beta_{2} & -\beta_{2} & 0
\end{array}\right], \\
\boldsymbol{B}_{\boldsymbol{\Gamma}}=\left[\begin{array}{c}
\frac{k_{1} b}{\bar{b}} \\
k_{1} \\
0
\end{array}\right], \boldsymbol{C}_{\boldsymbol{\Gamma}}=\left[\begin{array}{lll}
1 & 0 & 0
\end{array}\right] .
\end{gathered}
$$

In the same way, for SOPTD system, we can derived that

$$
\begin{gathered}
\boldsymbol{\Gamma}^{\prime}=\left[\begin{array}{l}
x_{1} \\
x_{2} \\
z_{1} \\
z_{2} \\
z_{3}
\end{array}\right], \boldsymbol{r}^{\prime}(t)=\left[\begin{array}{l}
r(t) \\
\dot{r}(t)
\end{array}\right], \quad \boldsymbol{B}_{\boldsymbol{\Gamma}}^{\prime}=\left[\begin{array}{cc}
0 & 0 \\
\frac{k_{1} b}{\bar{b}} & \frac{k_{2} b}{\bar{b}} \\
0 & 0 \\
k_{1} & k_{2} \\
0 & 0
\end{array}\right], \\
\boldsymbol{A}_{\boldsymbol{\Gamma}}^{\prime}=\left[\begin{array}{ccccc}
0 & 1 & 0 & 0 & 0 \\
-a_{0} & -a_{1} & -\frac{k_{1} b}{\bar{b}} & -\frac{k_{2} b}{\bar{b}} & -\frac{b}{\bar{b}} \\
\beta_{1} & 0 & \beta_{1} & 1 & 0 \\
\beta_{2} & 0 & -k_{1}-\beta_{2} & k_{2} & 0 \\
\beta_{3} & 0 & -\beta_{3} & 0 & 0
\end{array}\right], \\
C_{\boldsymbol{\Gamma}}^{\prime}=\left[\begin{array}{lllll}
1 & 0 & 0 & 0 & 0
\end{array}\right] .
\end{gathered}
$$

Then, we will prove the stability of the closed-loop system with FFC-ADRC design based on Lyapunov stability and the theory of input-output stability.

Definition 1 Lyapunov stability [37] For the autonomous system $\dot{\boldsymbol{X}}(t)=f(\boldsymbol{X}, t)$ with $\boldsymbol{X}\left(t_{0}\right)=\boldsymbol{X}_{0}$ and $t \in\left[t_{0}, \infty\right)$, the balanced state $\boldsymbol{X}_{e}=0$ is Lyapunov stability, if there is a real number $\delta\left(\varepsilon, t_{0}\right)>0, \forall \varepsilon>0$ such that $\left\|\boldsymbol{X}\left(t ; x_{0}, t_{0}\right)-\boldsymbol{X}_{e}\right\| \leqslant \varepsilon$ when $\left\|\boldsymbol{X}_{0}-\boldsymbol{X}_{e}\right\| \leqslant \delta\left(t=t_{0}\right)$ is established, where $\boldsymbol{X}\left(t ; \boldsymbol{X}_{0}, t_{0}\right)$ is the corresponding state response of the given $\boldsymbol{X}_{0}$.

Definition 2 Bound input bound output (BIBO) stability [37] For a system, giving any bounded input, i.e. $|r(t)| \leqslant \xi<\infty, \xi \geqslant 0$, if the corresponding system output is bounded, i.e. $\left|y_{\Gamma}(t)\right| \leqslant \varepsilon<\infty \varepsilon \geqslant 0, \forall t \in[0,+\infty)$, then the system is BIBO stability.

Lemma 1 [37] For a continuous linear system, if the system is observable and controllable, combing with Definition 1 , the system is asymptotic stable.

Theorem 1 For the time-delay system (1) and (2), the closed-loop system controlled via FFC-ADRC is asymptotic stability. The control law is given by (18) and (19), and the modified ESO is given by (17). Furthermore, the stability is related with $k_{i}, \beta_{i}, \bar{b}$.

Proof Solving (30), we can obtain that

$$
\boldsymbol{\Gamma}(t)=\boldsymbol{\Phi}(t) \boldsymbol{\Gamma}\left(t_{0}\right)+\int_{0}^{t} \boldsymbol{\Phi}(t-\tau) \boldsymbol{B}_{\boldsymbol{\Gamma}} r(\tau) \mathrm{d} \tau
$$

where $\boldsymbol{\Phi}(t)$ is the state-transition matrix, and $\boldsymbol{\Gamma}\left(t_{0}\right)$ is the original state.

According to Definition 1, considering the first part on the right side of (31), let $\boldsymbol{\Gamma}_{0}(t)=\boldsymbol{\Phi}(t) \boldsymbol{\Gamma}\left(t_{0}\right)$. The balanced state of the closed-loop system (30) is zero, i.e. $x_{e}=0$.

From (30), it can be guaranteed that $\boldsymbol{A}_{\boldsymbol{\Gamma}}$ is Hurwitz with proper parameters $k_{i}, \beta_{i}, \bar{b}$, and

$$
\boldsymbol{A}_{\Gamma}^{-1}=\left[\begin{array}{ccc}
0 & -\frac{1}{k_{1}} & \frac{k_{1}+\beta_{1}}{k_{1} \beta_{2}} \\
0 & -\frac{1}{k_{1}} & \frac{\beta_{1}}{k_{1} \beta_{2}} \\
-\bar{b} & \frac{a \bar{b}+b k_{1}}{b k_{1}} & -\frac{a \bar{b} k_{1}+a \bar{b} \beta_{1}+b k_{1} \beta_{1}}{b k_{1} \beta_{2}}
\end{array}\right],
$$

so it can be derived that

$$
\left|(\boldsymbol{\Phi}(t))_{i j}\right| \leqslant \varphi, \exists t>T_{1}, i, j=1,2,3
$$

where $\varphi$ is relative with $k_{1}, \beta_{1}, \beta_{2}, \bar{b}$. Hence, with an arbitrary original state $\boldsymbol{\Gamma}\left(t_{0}\right)$ satisfying that $\left\|\boldsymbol{\Gamma}\left(t_{0}\right)-\boldsymbol{X}_{e}\right\| \leqslant$ $\delta\left(t=t_{0}\right)$, one can obtain that

$$
\left\|\boldsymbol{\Gamma}_{0}(t)-\boldsymbol{X}_{e}\right\|=\left\|\boldsymbol{\Phi}(t) \boldsymbol{\Gamma}\left(t_{0}\right)\right\| \leqslant \varphi \delta .
$$

Defining $\varepsilon \triangleq \varphi \delta$, thus $x_{e}$ is Lyapunov stable.

Then considering the second part on the right side of (31), let $\boldsymbol{\Psi}(t)=\int_{0}^{t} \boldsymbol{\Phi}(t-\tau) \boldsymbol{B}_{\Gamma} r(\tau) d \tau$. Assuming $r(t)$ is bounded, i.e. $|r(t)| \leqslant \xi(x i \geqslant 0)$

$$
|\boldsymbol{\Psi}(t)|=\left|\int_{0}^{t} \boldsymbol{\Phi}(t-\tau) \boldsymbol{B}_{\Gamma} r(\tau) \mathrm{d} \tau\right| \leqslant
$$




$$
\begin{gathered}
\left|\xi \int_{0}^{t} \boldsymbol{\Phi}(t-\tau) \mathrm{d} \tau \boldsymbol{B}_{\boldsymbol{\Gamma}}\right|= \\
\left|\xi\left(\boldsymbol{A}_{\boldsymbol{\Gamma}}^{-1} \boldsymbol{\Phi}(t) \boldsymbol{B}_{\boldsymbol{\Gamma}}-\boldsymbol{A}_{\boldsymbol{\Gamma}}^{-1} \boldsymbol{B}_{\boldsymbol{\Gamma}}\right)\right| .
\end{gathered}
$$

According to $|a-b| \leqslant|a|+|b|$, (34) can be rewritten as

$$
\left|\boldsymbol{\Psi}_{i}(t)\right| \leqslant \xi\left[\left|\left(\boldsymbol{A}_{\boldsymbol{\Gamma}}^{-1} \boldsymbol{\Phi}(t) \boldsymbol{B}_{\boldsymbol{\Gamma}}\right)_{i}\right|+\left|\left(\boldsymbol{A}_{\boldsymbol{\Gamma}}^{-1} \boldsymbol{B}_{\boldsymbol{\Gamma}}\right)_{i}\right|\right] .
$$

Combing with (32), one can obtain that

$$
\left|\left[\boldsymbol{\Phi}(t) \boldsymbol{B}_{\boldsymbol{\Gamma}}\right]_{i}\right| \leqslant \chi,\left|\left[\boldsymbol{A}_{\boldsymbol{\Gamma}}^{-1} \boldsymbol{\Phi}(t) \boldsymbol{B}_{\boldsymbol{\Gamma}}\right]_{i}\right| \leqslant \chi \zeta
$$

where $t>T_{1}, \chi=\left(\frac{k_{1} b}{\bar{b}}+k_{1}\right) \varphi$, and

$$
\begin{gathered}
\zeta=\max \left\{\left|\frac{\beta_{1}+k_{1}-\beta_{2}}{k_{1} \beta_{2}}\right|,\right. \\
\left.\left|\frac{b k_{1} \beta_{2}+a \bar{b} \beta_{2}-\bar{b} k_{1} \beta_{2}-a \bar{b} k_{1}-a \bar{b} \beta_{1}-b k_{1} \beta_{1}}{b k_{1} \beta_{2}}\right|\right\} .
\end{gathered}
$$

Then according to $\boldsymbol{A}_{\boldsymbol{\Gamma}}^{-1}$ and $\boldsymbol{B}_{\boldsymbol{\Gamma}}$ defined above, it yields that

$$
\boldsymbol{A}_{\Gamma}^{-1} \boldsymbol{B}_{\boldsymbol{\Gamma}}=\left[\begin{array}{c}
-1 \\
-1 \\
a \frac{b}{b}
\end{array}\right] .
$$

Therefore, we can derive that

$$
\left|\left(\boldsymbol{A}_{\boldsymbol{\Gamma}}^{-1} \boldsymbol{B}_{\boldsymbol{\Gamma}}\right)_{i}\right| \leqslant \nu, \quad \nu=\max \left\{1,\left|a \frac{\bar{b}}{b}\right|\right\} .
$$

Combining (33), (34), (35) and (38), we can conclude that

$$
\begin{gathered}
\left|\boldsymbol{\Gamma}_{i}(t)\right|=\left|\boldsymbol{\Gamma}_{0}(t)_{i}\right|+\left|\boldsymbol{\Psi}_{i}(t)\right| \leqslant \\
\varphi \delta+\xi(\chi \zeta+\nu), t>T_{1} .
\end{gathered}
$$

Thus according to (39) and $y_{\Gamma}(t)=\left[\begin{array}{lll}1 & 0 & 0\end{array}\right] \boldsymbol{\Gamma}(t)$, we have

$$
\left|y_{\Gamma}(t)\right| \leqslant \varphi \delta+\xi(\chi \zeta+\nu) \triangleq \sigma
$$

for $t>T_{1}$. We can obtain the same conclusion for the SOPTD system in similar way. Therefore, according to Definition 2, the closed-loop system controlled via FFCADRC is BIBO stability.

In addition, the closed-loop system (30) is observable and controllable with appropriate parameters, i.e. $\operatorname{rank}\left[\boldsymbol{B}_{\boldsymbol{\Gamma}}, \boldsymbol{A}_{\boldsymbol{\Gamma}} \boldsymbol{B}_{\boldsymbol{\Gamma}}, \boldsymbol{A}_{\boldsymbol{\Gamma}}^{2} \boldsymbol{B}_{\boldsymbol{\Gamma}}\right]=3, \operatorname{rank}\left[\boldsymbol{C}_{\boldsymbol{\Gamma}}, \boldsymbol{A}_{\boldsymbol{\Gamma}} \boldsymbol{C}_{\boldsymbol{\Gamma}}\right.$, $\left.\boldsymbol{A}_{\boldsymbol{\Gamma}}^{2} \boldsymbol{C}_{\boldsymbol{\Gamma}}\right]^{\mathrm{T}}=3$.

According to Lemma 1, the closed-loop system is asymptotic stable. Furthermore, since $\varphi, \zeta, \nu$ and $\chi$ are related with $k_{1}, \beta_{1}, \beta_{2}, \bar{b},\left|y_{\Gamma}(t)\right| \leqslant \sigma$ and $\sigma$ is related with $k_{i}, \beta_{i}, \bar{b}$.

Remark 2 In a continuous linear system, if it is Lyapunov stable and the zero-state response is bounded with any bounded input, the system is BIBO stability. Furthermore, if it is also controllable and observable, the system is asymptotic stable (or global asymptotic stable).

\subsection{Parameter tuning rules}

In the FFC-ADRC design, $k_{i}, \beta_{i}$ and $\bar{b}$ need to be tuned. Similar to traditional ADRC, we translate $k_{i}, \beta_{i}$ into expressions relating with $\omega_{o}$ and $\omega_{c}[31] ; \bar{b}$ should be tuned around $b$, and it will be larger with the increase of time delay $\tau$ [36]. The gain $k_{1}$ cannot be too large, because a large $k_{1}$ will lead to system oscillation. Then the detail procedures of parameter tuning are as follows:

(i) If the model is known, the value of $\tau_{0}$ and $\bar{b}$ can be selected according to $\tau$ and $b$; or else they can be tuned based on the above analysis.

(ii) Select $1 / \tau$ as the initial value of $\omega_{c}$ [6], and the initial $\omega_{o}$ can be $2-10$ times higher than the controller bandwidth.

(iii) Increase the value of $\omega_{o}$ gradually with keeping $\omega_{c}$ constant until the noise impact is difficult to meet system requirements. In the same way, keeping the $\omega_{o}$ constant, increase $\omega_{c}$ until the system output fluctuates; then decrease $\omega_{o}$ and increase $\omega_{c}$ continually. According to that way, tune $\omega_{c}$ and $\omega_{o}$ until meet control requirements.

(iv) If it has much fluctuation in the tracking process, we should tune the value of $\bar{b}$.

Normally the higher the bandwidth is, the better the performance is. While the cost is that the system is susceptible to the noise. Thus, when the information about time delay systems is not exactly known, the bandwidth cannot be too large.

\section{Simulation analysis}

In order to demonstrate the numerical behavior of the FFCADRC design, we present a controller design solution for the FOPTD system. Simulation comparisons between the widely used SP with proportional-integral (PI) controller and FFC-ADRC are shown in Section 4.1. Furthermore, Section 4.2 presents a test for the SOPTD system to illustrate the universality of the method proposed in this paper.

\subsection{Simulation analysis of FOPTD system}

As for the FOPTD system, $a=10, b=1, \tau=5 \mathrm{~s}$, based on the parameter tuning discussed above, parameters of FFC-ADRC are selected as follows: $\omega_{o}=50, \omega_{c}=9$, $\bar{b}=1.5$. As a comparison, an SP with PI controller (SPPI) is tuned as $k_{p}=12, k_{i}=30$.

Firstly, the step response is shown in Fig. 5. An external disturbance is injected at $50 \mathrm{~s}$ with the final value 0.5 simultaneously. 


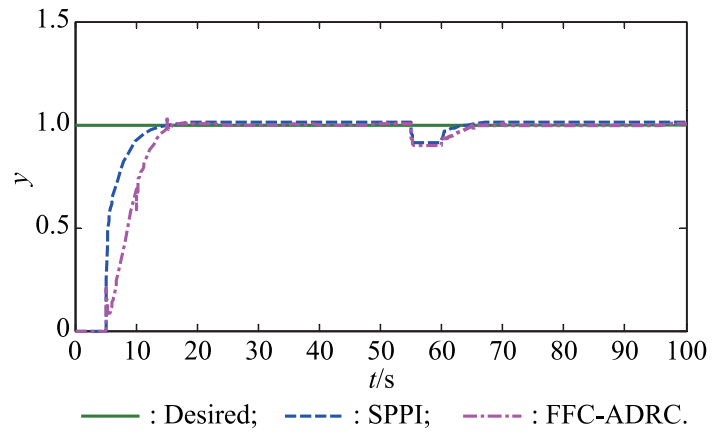

Fig. 5 Step response of closed-loop system

From Fig. 5, the performance of SPPI and FFC-ADRC is very close because of the accurate model information.

Secondly, to test the robustness of the proposed method, a system parameter perturbation is added while maintaining parameters of the controller constant, as shown in Fig. 6.

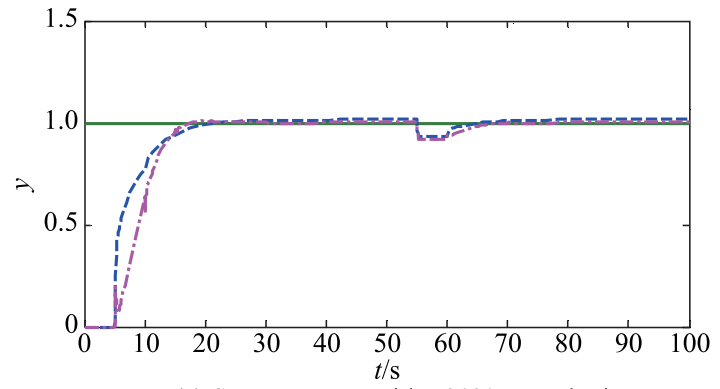

(a) Step response with $+20 \%$ perturbation

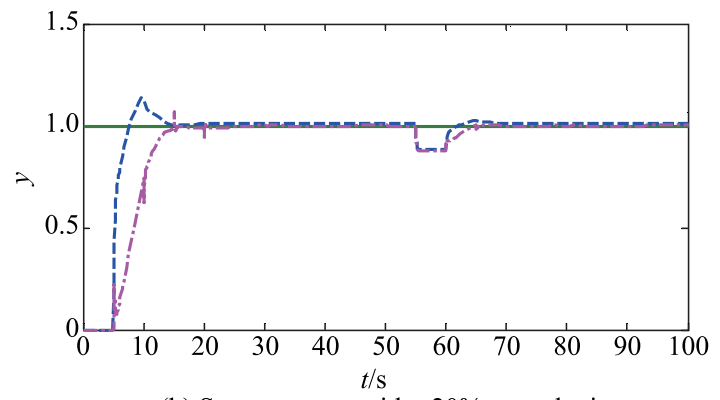

(b) Step response with $-20 \%$ perturbation

—— : Desired; ---- : SPPI; ---.- : FFC-ADRC.

Fig. 6 Step response with $\pm \mathbf{2 0} \%$ perturbation

In Fig. 6, with parameter perturbation of $+20 \%$, the settling time of SPPI controller is about $25 \mathrm{~s}$, and the settling time of FFC-ADRC is about $18 \mathrm{~s}$; with parameter perturbation of $-20 \%$, there is a big overshoot controlled by SPPI, and the anti-disturbance ability is worse than FFC-ADRC obviously after adding a disturbance. Thus, this demonstrates the advantage of the proposed method over SPPI in terms of the robustness.

Furthermore, a perturbation of $\pm 20 \%$ is added to the time delay while keeping controller parameters constant, as shown in Fig. 7.

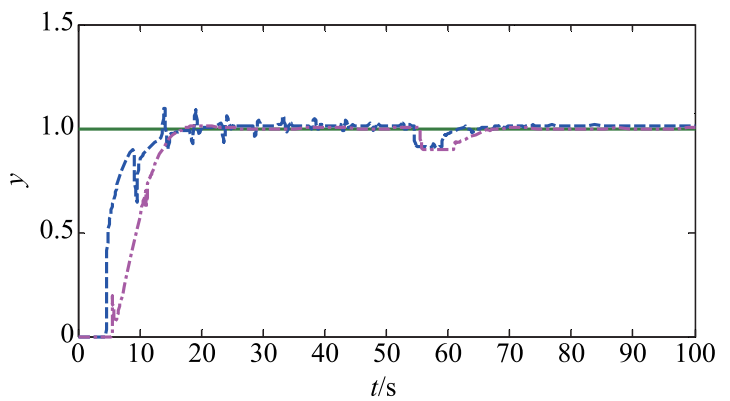

(a) Step response with $+20 \%$ delay

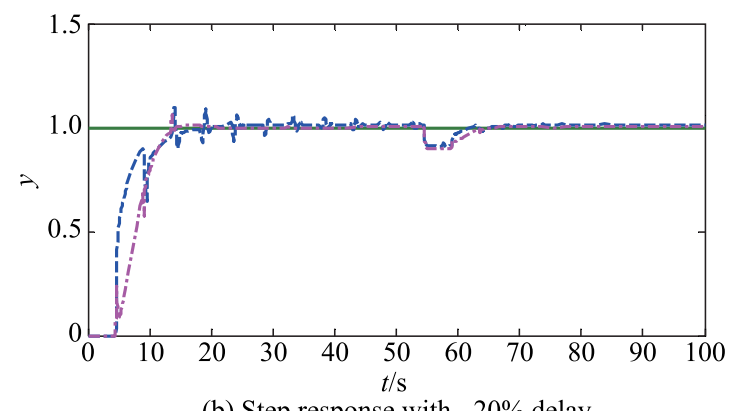

(b) Step response with $-20 \%$ delay

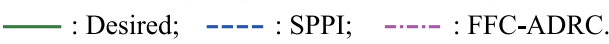

Fig. 7 Step response with $\pm 20 \%$ delay

In Fig. 7, the SPPI controller has a big oscillation and a bad anti-disturbance capability. And it may be unstable when giving a bigger time perturbation. Obviously, the FFC-ADRC controller can reject this disturbance well. Therefore, we can conclude that the SPPI controller is sensitive to the accuracy of the system model, while FFCADRC has a stronger robustness due to own advantages of ADRC.

Then to test that FFC-ADRC can control the time-delay system with positive, zero and negative poles, numerical simulations are carried out. The unit step response with different poles is shown in Fig. 8.

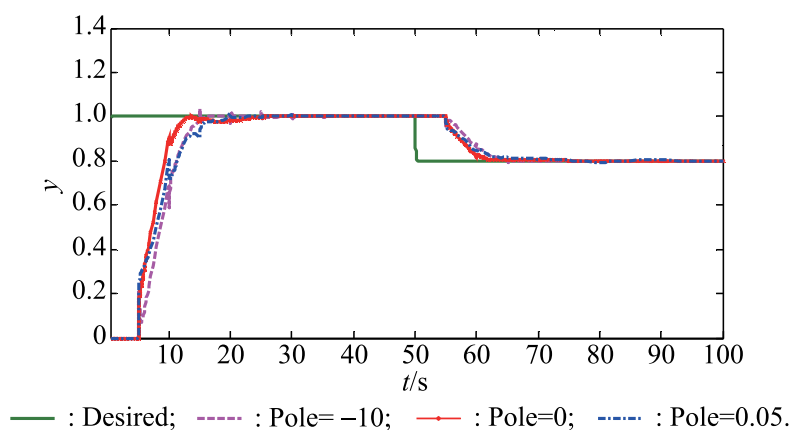

Fig. 8 FFC-ADRC for stable, critical stable and unstable system

From Fig. 8, FFC-ADRC can control the stable, critical stable and unstable systems with time delay well.

Finally, in order to further test the generality of the proposed design, we take simulations about systems with 
$a=1, b=1, \tau=0.3 \mathrm{~s}$ and $\tau=2 \mathrm{~s}$, respectively. According to Section 2.1, the control difficulty is $\theta=0.3$ and $\theta=2$, respectively. Fig. 9 and Fig. 10 show responses with a different perturbation of the time delay.

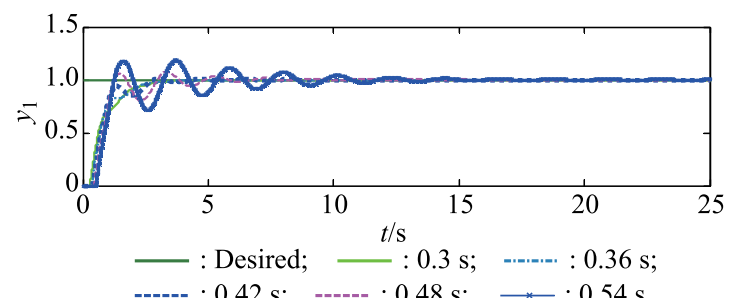

(a) $y_{1}$

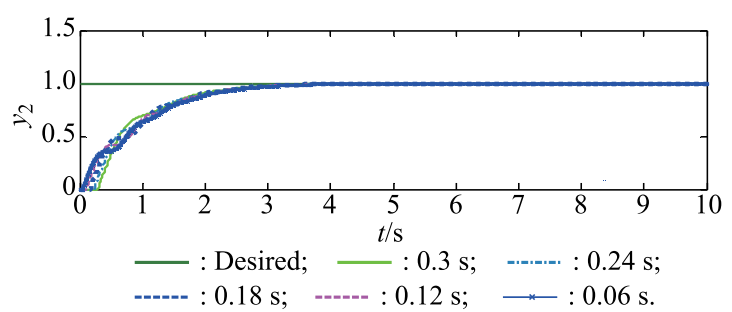

(b) $y_{2}$

Fig. 9 Step response with $\theta=0.3$

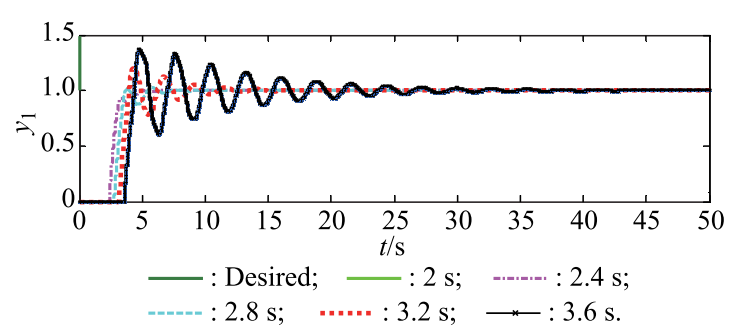

(a) $y_{1}$

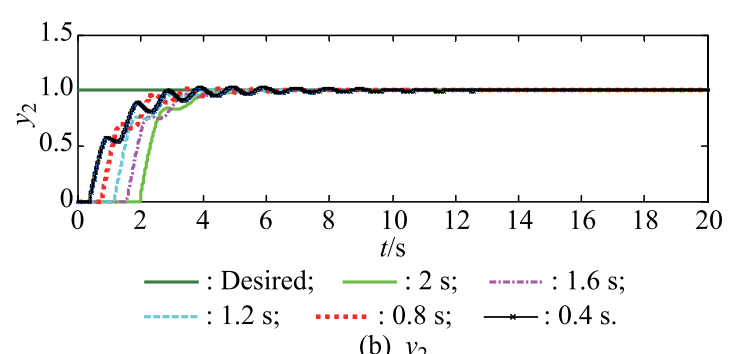

(b) $y_{2}$

Fig. 10 Step response with $\theta=2$

In Fig. 9 and Fig. 10, we add time-delay perturbations of $\pm 20 \%, \pm 40 \%, \pm 60 \%$ and $\pm 80 \%$, respectively. As we can see from the result, with the increase of the time-delay perturbation, the settling time becomes longer; and the oscillation appears obviously when adding time-delay perturbations of $\pm 60 \%$ and $\pm 80 \%$. Therefore, we can conclude that FFC-ADRC has a much larger stability margin and keeps the system under control for up to $\pm 40 \%$ of the time-delay mismatch.
According to results above all, we can conclude that FFC-ADRC is a good solution for systems with time delay.

\subsection{Simulation analysis of SOPTD system}

In this part, we will carry out the test on the SOPTD system by using FFC-ADRC design in order to verify the universality of FFC-ADRC. The SOPTD is given by

$$
G(s)=\frac{2}{(3 s-1)(s+1)} \mathrm{e}^{-0.3 s} .
$$

A second-order FFC-ADRC is designed with parameters of $\omega_{o}=16, \omega_{c}=10, \bar{b}=1.2$. The step response with a perturbation of $\tau$ is shown in Fig. 11. An external disturbance with a magnitude of 0.5 is injected at $10 \mathrm{~s} \mathrm{si-}$ multaneously.

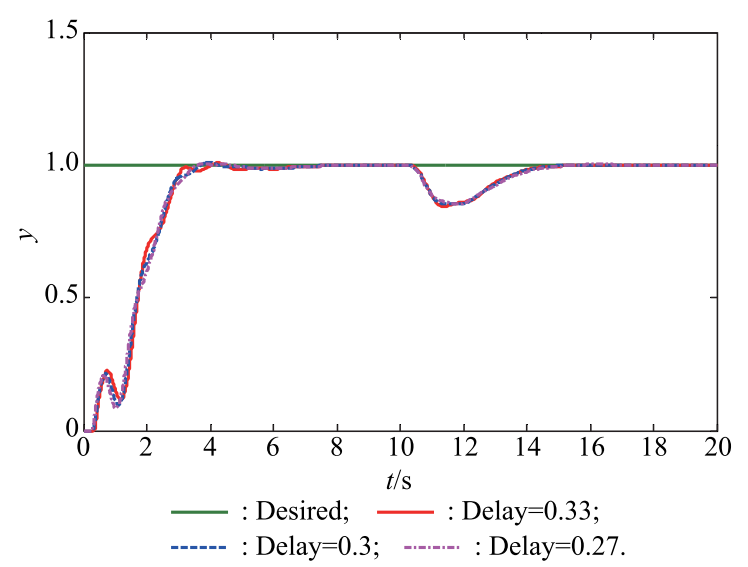

Fig. 11 Step response of second-order closed-loop system with $\tau=$ $0.33,0.3,0.27$

When added a parameter perturbation of $\pm 20 \%$, the step response is shown in Fig. 12.

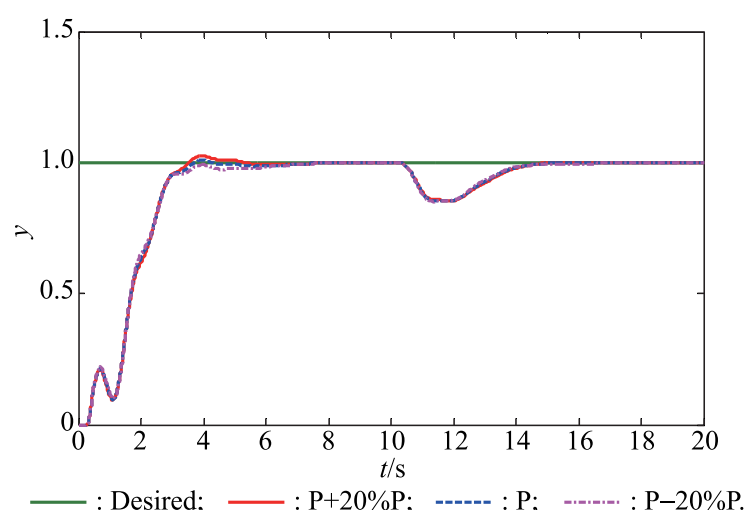

Fig. 12 Step response of second-order closed-loop system with parameter perturbation

In Fig. 11 and Fig. 12, FFC-ADRC can control the SOPTD system well and has a strong anti-disturbance ability and robustness. 
Based on all the above analysis, we can conclude that the proposed method in this paper is effective and accurate for the time-delay system with stable, critical stable or unstable poles.

\section{Conclusions}

This paper mainly proposes a novel method (FFC-ADRC) for a class of input time-delay systems with stable, critical stable or unstable poles. FFC-ADRC is designed based on the idea of the SP method and the traditional ADRC to synchronize the control signal and the output signal. It improves the disturbance rejection performance over the traditional ADRC design by increasing the achievable observer bandwidth. Owing to the ability of ADRC to deal with internal uncertainties, the proposed design also turns out to be a unified solution for time-delay systems with stable, critical stable and unstable poles. And then the asymptotic stability of closed-loop system is verified. Meanwhile, the procedure of the parameter tuning is discussed. Finally, numerical examples show that FFC-ADRC can reduce the influence led by time delay. Significant improvements over existing methods are observed.

\section{References}

[1] H. J. Gao, T. W. Chen, J. Lam. A new delay system approach to network-based control. Automatica, 2008, 44(1): 39-52.

[2] D. Enns, H. Ozbay, A. Tannenbaum. Abstract model and controller design for an unstable aircraft. Journal of Guidance, Control \& Dynamics, 2015, 15(2): $498-508$.

[3] D. E. Seborg, T. F. Edgar, D. A. Mellichamp. Process dynamics and control-modeling for control and prediction. New Jersey: Wiley, 2004.

[4] J. P. Richard. Time-delay systems: an overview of some recent advances and open problems. Automatica, 2003, 39(10): $1667-1694$.

[5] P. Garcła, P. Albertos, T. Hägglund. Control of unstable nonminimum-phase delayed systems. Journal of Process Control, 2006, 16(10): 1099-1111.

[6] S. Skogestad, I. Postlethwaite. Multivariable feedback control: analysis and design. New York: Wiley, 1996.

[7] G. J. Silva, A. Datta, S. P. Bhattacharyya. New results on the synthesis of PID controllers. IEEE Trans. on Automatic Control, 2002, 47(2): 241-252.

[8] M. Ge, M. S. Chiu, Q. G. Wang. Robust PID controller design via LMI approach. Journal of Process Control, 2002, 12(1): $3-13$.

[9] G. J. Silva, A. Datta, S. P. Bhattacharyya. PID controllers for time-delay systems. Basel: Birkhäuser Boston, 2005.

[10] A. Manitius, A. W. Olbrot. Finite spectrum assignment problem for systems with delays. IEEE Trans. on Automatic Control, 1979, 24(4): $541-552$.

[11] E. Furutani, T. Hagiwara, M. Araki. Two-degree-of-freedom design method of state-predictive LQI servo systems. IEE Proceedings of Control Theory and Applications, 2002, 149(5): $365-378$.

[12] O. J. M. Smith. Closed control of loops with dead time. Chemical Engineering Progress, 1957, 53: 217 - 219.
[13] M. Mahmoud, P. Shi. Robust stability, stabilization and Hinfinity control of time-delay systems with Markovian jump parameters. International Journal of Robust and Nonlinear Control, 2003, 13(8): $755-784$

[14] M. R. Matausek, A. D. Micic. A modified Smith predictor for controlling a process with an integrator and long dead-time. IEEE Trans. on Automatic Control, 1996, 41(8): 1199-1203.

[15] T. Liu, W. D. Zhang, D. Y. Gu. Analytical design of twodegree-of-freedom control scheme for open-loop unstable processes with delay. Journal of Process Control, 2005, 15(5): $559-572$.

[16] J. E. Normey-Rico, E. F. Camacho. Dead-time compensators: A survey. Control Engineering Practice, 2008, 16(4): $407-$ 428.

[17] P. García, P. Albertos. Robust tuning of a generalized predictor based controller for integrating and unstable systems with long time delay. Journal of Process Control, 2013, 23(8): $1205-$ 1216.

[18] B. Chen, C. Lin, X. P. Liu, et al. Observer-based adaptive fuzzy control for a class of nonlinear delayed systems. IEEE Trans. on Systems, Man and Cybernetics: Systems, 2016, 46(1): $27-$ 36.

[19] H. H. Wang, J. C. Liu, F. S. Yang, et al. Proportional-integral controller for stabilization of second-order delay processes. International Journal of Control, Automation \& Systems, 2014, 12(6): $1197-1206$.

[20] M. Q. Liu, H. Y. Chen, S. L. Zhang, et al. $\mathrm{H} \infty$ synchronization of two different discrete-time chaotic systems via a unified model. International Journal of Control, Automation \& Systems, 2015, 13(1): 212-221.

[21] S. J. Yoo. Delay-independent fault detection and accommodation for non-linear strict-feedback systems with unknown time-varying delays. IET Control Theory \& Applications, 2015, 9(2): 293-299.

[22] N. T. Mai, T. D. Vu, S. Radziah, et al. A design method for modified smith predictors for multiple-input/multiple-output non-minimum-phase time-delay plants with feedback connected multiple time-delays. Proc. of the 10th Asian Control Conference, 2015: 1-4.

[23] L. Wang, Q. Li, C. Tong, et al. On control design and tuning for first order plus time delay plants with significant uncertainties. Proc. of American Control Conference, 2015: 5276-5281.

[24] J. Q. Han. Active disturbance rejection control technique: the technique for estimating and compensating the uncertainties. Beijing: National Defense Industry Press, 2008. (in Chinese)

[25] Y. Huang, W. C. Xue. Active disturbance rejection control: methodology and theoretical analysis. ISA Transactions, 2014, 53(4): $963-976$

[26] M. Ramírez-Neria, H. Sira-Ramírez, R. Garrido-Moctezuma, et al. Linear active disturbance rejection control of underactuated systems: the case of the Furuta pendulum. ISA Transactions, 2014, 43(4): 920-928.

[27] R. Madoński, M. Kordasz, P. Sauer. Application of a disturbance-rejection controller for robotic-enhanced limb rehabilitation trainings. ISA Transactions, 2014, 53(4): 899908.

[28] Y. Q. Xia, L. Dai, M. Y. Fu, et al. Application of active disturbance rejection control in tank gun control system. Journal of the Franklin Institute, 2014, 351(4): 2299-2314.

[29] M. S. Fadali. On the stability of Han's ADRC. Proc. of American Control Conference, 2014: 3597-3601.

[30] B. Z. Guo, Z. L. Zhao. On the convergence of an extended state observer for nonlinear systems with uncertainty. Systems and 
Control Letters, 2011, 60: 420-30.

[31] Z. Q. Gao. Scaling and bandwidth-parameterization based controller tuning. Proc. of American Control Conference, 2003: $4989-4996$.

[32] J. Q. Han, W. G. Zhang. ADRC control for large time-delay systems. Control and Decision, 1999, 14(4): 354-358. (in Chinese)

[33] Y. Q. Xia, P. Shi, G. P. Liu, et al. Active disturbance rejection control for uncertain multivariable systems with time-delay. IET Control Theory and Applications, 2007, 1(1): 75 -81.

[34] Y. Q. Xia, M. Y. Fu, P. Shi. Analysis and synthesis of dynamical systems with time delays. Berlin: Springer Science and Business Media, 2009.

[35] Q. L. Zheng, Z. Gao. Predictive active disturbance rejection control for processes with time delay. ISA Transactions, 2013, 53(4): $873-881$.

[36] J. Q. Han. Auto-disturbances rejection control for time-delay systems. Control Engineering of China, 2008, 15(S1): 7-11. (in Chinese)

[37] D. Z. Zheng. Linear system theory. Beijing: Tsinghua University Press, 1990. (in Chinese)

\section{Biographies}

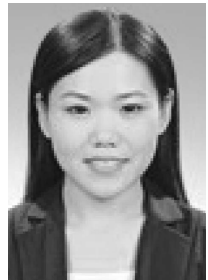

Dongyang Zhang was born in 1987. She is a Ph.D. candidate in control theory and control engineering, Beijing Institute of Technology. She received her B.S. degree in measuring and controlling technology and instrument, from Yanshan University in 2011. She received her M.S. degree in control engineering from Beijing Institute of Technology in 2013. Her research interest covers control of industry and active disturbance rejection control.

E-mail: zhangdongyang2006@163.com

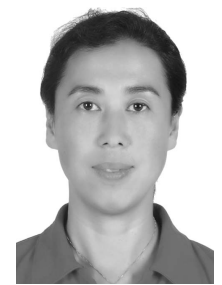

Xiaolan Yao was born in 1964. She received her B.S. degree in mechanical engineering in 1987, M.S. degree in automatic control in 1990 and Ph.D. degree in electromechanical engineering in 2003, respectively, from Beijing Institute of Technology. She is a professor in Beijing Institute of Technology. Her research interest covers complicated industry system control.

E-mail: yaoxiaolan@bit.edu.cn

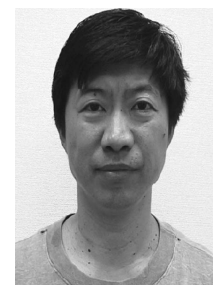

Qinghe Wu was born in 1954. He received his B.S. degree in automatic control, in 1982, from Central College of Engineering, Huazhong University of Science and Technology, and M.S. degree and Ph.D. degree in 1984 and 1990, respectively, from Eidgenössische Technische Hochsohule Zurich (ETH). $\mathrm{He}$ is a professor in Beijing Institute of Technology. His research interest covers control theory and robustness control.

E-mail: qinghew@bit.edu.cn

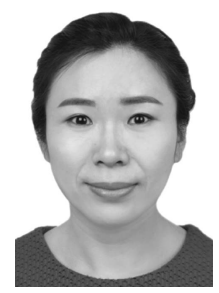

Zhuoyue Song was born in 1982 . She received her B.S. degree in electrical engineering and automation from Hebei University of Science and Technology in 2004, M.S. degree in control science and engineering from Harbin University of Technology in 2007 (with first class honours) and Ph.D. degree in electrical and electronic engineering from University of Manchester in 2011. She is currently a lecturer in the School of Automation at Beijing Institute of Technology, Beijing, China. Her research interests include analysis and synthesis of control systems, with particular attention to uncertainty, optimization and multi-agent systems.

E-mail: szhuoyue@bit.edu.cn 